\title{
Improving space-time code performance in slow fading channels using reconfigurable antennas
}

\author{
Philippa A. Martin, IEEE Senior Member, Peter J. Smith, IEEE Senior Member and Ross Murch, IEEE Fellow
}

\begin{abstract}
Space-time block codes typically only span a small number of time slots. However, error control coding spanning hundreds or thousands of symbol periods is often used with space-time coding. In this case, slow fading channels significantly degrade performance. In this letter, we show that performance can be significantly improved in slow fading conditions by using reconfigurable antennas. We propose switching the transmit antenna states during transmission so that the error control code experiences several different channel conditions over the transmission period. Hence, it can achieve higher diversity and the superior performance expected of a faster fading channel due to reconfigurable antenna state switching. If we also allow the receiver to select the reconfigurable receive antenna states based on channel energy, then an antenna state selection gain is also achieved.
\end{abstract}

\section{INTRODUCTION}

Reconfigurable antennas are able to change the manner in which they radiate, by altering properties such as frequency, polarization and radiation pattern [2], producing different radiation states. Changing the radiation state also changes the channel characteristics experienced in the communication system [2]. In [2], it was shown that reconfigurable antennas can be used to improve the performance of orthogonal spacetime block coded transmission over quasi-static flat Rayleigh fading channels. Then, in [1], the reconfigurable antenna states were used as a dimension in designing space-time-state block coded multiple-input multiple-output (MIMO) systems.

Space-time block codes typically span only a small number of time slots. They are usually simulated assuming quasi-static channel models, which are constant over the length of the space-time code and vary independently between codewords. However, error control codes spanning hundreds or thousands of symbol periods are often used in conjunction with spacetime coding. Now, the quasi-static channel spans the length of the error control code and corresponds to much slower fading. Slow fading channels significantly degrade performance [6]. Alternatively, the channel could be modeled to change independently for every space time codeword, but this implies the use of a large channel interleaver employed over multiple error control codewords, which is unattractive from a practical viewpoint.

In this letter, we show that the degradation in performance caused by slow fading can be significantly reduced by using reconfigurable antennas. We propose changing the antenna

Dr. Martin and Assoc. Prof. Smith are with the Department of Electrical and Computer Engineering, University of Canterbury, New Zealand. \{philippa.martin, peter.smith\}@canterbury.ac.nz. Prof. Murch is with the Department of Electronic and Computer Engineering at the Hong Kong University of Science and Technology, Hong Kong. eermurch@ee.ust.hk. states (antenna state switching) during transmission in order to improve the performance of long space-time codes or MIMO transmissions using long outer error control codes. This allows the code to experience several different channel conditions over the transmission period. Note that antenna state switching is used in a predefined manner to alter the channel rather than to increase SNR. As a result, the system achieves higher diversity and the performance expected in much faster fading conditions. In addition, we investigate how selecting the antenna state based on the channel energy can provide signal to noise ratio (SNR) gains. From a practical viewpoint, we will show that the combination of antenna state switching at the transmitter and antenna state selection at the receiver is attractive.

This letter is organized as follows. Section II describes the space-time system considered. Section III proposes both antenna state selection and antenna state switching schemes for space-time coded systems. Simulation results are also included in Section III. Finally, conclusions are drawn in Section IV.

\section{SYSTEM DESCRIPTION}

We consider space-time systems with $N_{t}$ transmit antennas and $N_{r}$ receive antennas. The $\left(1 \times N_{r}\right)$ received vector at time $t$ can be written as

$$
\boldsymbol{r}_{t}=\boldsymbol{s}_{t} \boldsymbol{H}_{t}+\boldsymbol{n}_{t}
$$

where $s_{t}$ is the $\left(1 \times N_{t}\right)$ transmitted vector at time $t$ and $\boldsymbol{n}_{t}$ is the $\left(1 \times N_{r}\right)$ additive white Gaussian noise (AWGN) vector at time $t$. The complex $N_{t} \times N_{r}$ channel matrix at time $t$ is denoted $\boldsymbol{H}_{t}$, where the element representing the subchannel from the $i^{\text {th }}$ transmit antenna to the $j^{\text {th }}$ receive antenna is denoted $h_{t, i, j}$. We assume that all transmit to receive antenna sub-channels are independent and that we have ideal channel state information (CSI) at the receiver, but none at the transmitter. There is no feedback path from receiver to transmitter unless otherwise stated.

Two flat Rayleigh fading channel models are used in this letter. We consider a temporally correlated fading channel, where the speed of the fading is defined by the normalized fade rate, $f_{D} T, f_{D}$ is the Doppler frequency and $T$ is the symbol period. A $3^{r d}$ order Butterworth filter with cutoff frequency $f_{D}$ is used to generate the fading gains, $h_{t, i, j}$, for each subchannel. We also consider an independent or ideally interleaved flat Rayleigh fading channel. In this case, the fading channel gains change independently in each time slot and each $h_{t, i, j}$ is a complex Gaussian random variable with zero mean and unit variance. This approximates very fast fading or a system 
with sufficient interleaving to result in temporally uncorrelated received symbols.

For simplicity, we consider a direct transmission scheme [5], [6] where a low density parity check (LDPC) code is modulated onto 16-QAM and the resulting signal is multiplexed across $N_{t}$ transmit antennas. We denote its code rate as $\mathcal{R}_{e c c}$. LDPC codes were chosen due to their good performance and the availability of a simple iterative decoding algorithm (belief propagation). However, many other classes of codes could be used. The LDPC codes used here are designed using the near optimal degree sequences for the AWGN channel based on techniques in [7]. Specifically, the codes are designed for maximum girth using a progressive edge growth algorithm [4], which ensures an upper triangular parity check matrix for linear time encoding. The decoder uses belief propagation [3] operating in the log-domain [8]. The maximum number of decoding iterations for the LDPC code is 100, although the LDPC decoder will terminate early if a codeword is found. A bit interleaver is used after the LDPC encoder.

Note that other space-time coded or MIMO systems could be used with the proposed reconfigurable approach. Gains are expected from systems with coding (error control and/ or space-time) spanning hundreds or thousands of symbol periods. For example, space-time trellis codes transmitted over slow fading channels should benefit from our approach.

Assuming $M$-ary signalling, the $1-\mathrm{D}$ noise variance is defined as [6]

$$
\sigma_{n}^{2}=\frac{N_{t} E_{s}}{2 \log _{2}(M) \mathcal{R}_{e c c} 10^{0.1 S N R}},
$$

where $S N R$ is the average signal to noise ratio $\left(E_{b} / N_{0}\right)$ in decibels $(d B), E_{s}$ is the average energy of a constellation point, $E_{b}$ is the average energy per data bit and $N_{0}$ is the 2-sided noise spectral density. The rate of the space-time code is then defined as $\mathcal{R}_{s t c}=\mathcal{R}_{e c c} N_{t}$, giving the number of 2-D data constellation points transmitted per time slot.

\section{RECONFIGURABLE ANTENNAS}

In this section, we investigate simple design guidelines for using reconfigurable antennas with space-time systems that have coded blocks spanning hundreds or thousands of symbol periods. We assume $S_{t}$ radiation states per transmit antenna and $S_{r}$ radiation states per receive antenna. We assume uncorrelated radiation states and antennas. We will look at both switching antenna radiation states and selecting antenna radiation states.

The simulation results presented use a binary $(16384,8192)$ LDPC code Gray mapped to 16-QAM across $N_{t}=4$ transmit antennas and $L=1024$ time slots. We consider a critically loaded system with $N_{r}=4$ receive antennas. This space-time LDPC (ST-LDPC) code uses the soft output list sphere decoder of [5] with a list size of 512. Performance of the ST-LDPC code degrades as the fade rate decreases [6].

\section{A. Antenna State Switching}

We use the term antenna state switching to mean that some or all antenna radiation states are switched in a predefined pattern without the need for any channel information.

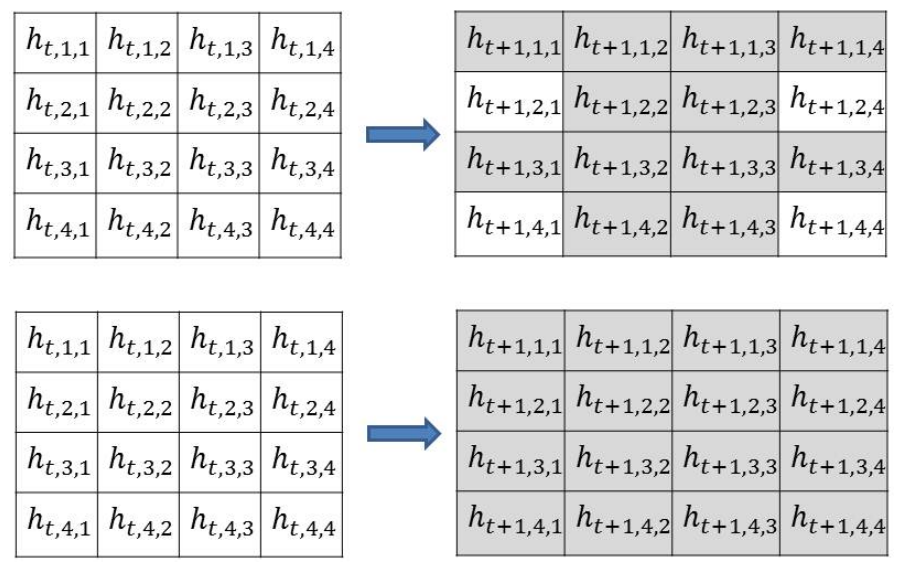

Fig. 1. Channel matrix changes (shaded squares) from antenna switching when $N_{t}=N_{r}=4$. In the top example we switch states for transmit antennas (rows) 1 and 3 , and for receive antennas (columns) 2 and 3 . As can be seen, there are still 4 matrix elements that are highly correlated with the corresponding previous channel matrix elements due to slow fading. If instead we switch the states of all transmit antennas or all receive antennas (bottom figure), then the whole channel matrix has changed independently from time $t$ to $t+1$

Switching could occur adaptively, when certain conditions are detected, but here we will use fixed switching times and a fixed pattern of states to switch between. In looking at antenna state switching, we begin by looking at how often we should switch antenna states and the gains possible.

In Fig. 1 we show the elements of the channel matrix that change when different antennas switch states (changes are denoted by shaded squares). If the state at transmit antenna $i$ or receive antenna $j$ is not switched and fading is slow, then $h_{t, i, j}$ and $h_{t+1, i, j}$ will be highly correlated. All values in $\boldsymbol{H}_{t}$ change if all transmit or all receive antenna states are changed at the same time as shown in Fig. 1. Therefore, this channel matrix will be independent from the previous one due to our channel assumptions. Since we assume uncorrelated antenna states and antennas, there are $S_{t} S_{r}$ independent channel matrices that can be generated from the original $\boldsymbol{H}_{t}$ by changing all transmit (row) or receive (column) antenna states. The total number of possible channel matrices is $P=S_{t}^{N_{t}} S_{r}^{N_{r}}$, labeled $\boldsymbol{H}_{t}(i)$, $i=1,2, \cdots, P$.

Let $L$ denote the number of symbol periods spanned by the error control code. Then, we can use a new independent channel matrix every

$$
T_{c_{\max }}=L /\left(S_{t} S_{r}\right)
$$

symbol periods. In [9], it was shown that symbols separated by $\beta$ symbol periods may be regarded as uncorrelated on SISO fading channels when

$$
\beta>\frac{0.3}{f_{D} T}
$$

This was used in [9] to choose the block interleaver length.

In Fig. 2, we can see the bit error rate (BER) performance as it changes with $f_{D} T$ for a ST-LDPC code using single state antennas, i.e. $S_{t}=S_{r}=1$. As seen in [9], performance improves with increased fade rate until approximately $f_{D} T=$ 


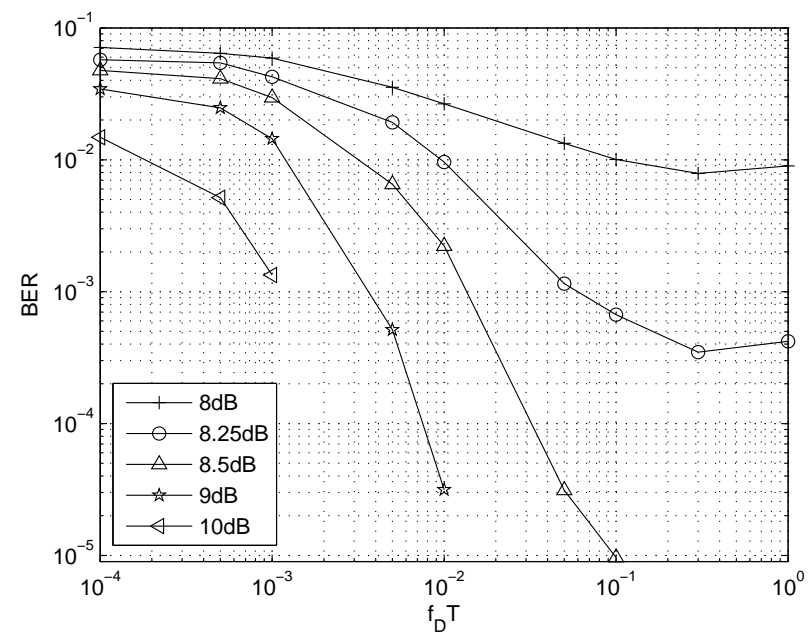

Fig. 2. BER versus $f_{D} T$ curves for a $\mathcal{R}_{s t c}=2$ ST-LDPC code using traditional antennas $\left(S_{t}=S_{r}=1\right)$ at $E_{b} / N_{0}=8, \cdots, 10 \mathrm{~dB}$. Each antenna transmits points from 16-QAM.

0.3. This validates the use of (4) in our MIMO reconfigurable antenna system design.

Say we are experiencing a fade rate $f_{D} T(1)$, but we want the performance of fade rate $f_{D} T(2)>f_{D} T(1)$. Here, we need

$$
T_{c_{\max }}<\frac{0.3}{f_{D} T(2)}
$$

which will give us a new channel every $T_{c_{\max }}$ symbol periods. Therefore, based on substituting (3) into (5), we propose choosing the values of $S_{t}$ and $S_{r}$ to satisfy

$$
S_{t} S_{r}>\frac{f_{D} T(2) L}{0.3} .
$$

In Fig. 3, we look at the effective fade rate, $f_{D} T(2)$, achieved by changing the channel matrix independently every $T_{c}$ symbol periods. Using $S_{t} S_{r}=4$ or $S_{t} S_{r}=16$ we can form an independent channel matrix every $T_{c}=L /\left(S_{t} S_{r}\right)=256$ or $T_{c}=64$ symbol periods, respectively, using antenna state switching every $T_{c}$ symbol periods. We show the performance for $f_{D} T=0.0001$ with the channel matrix changed independently every $T_{c}=1,64$ or 256 symbol periods. For comparison purposes, the performance of a ST-LDPC code using single state antennas $S_{t}=S_{r}=1$ is shown for various normalized fade rates, $f_{D} T$. We observe that the $f_{D} T=0.0001$ simulations with $T_{c}=256$ (and $\left.S_{t} S_{r}=4\right)$ and $T_{c}=64$ (and $S_{t} S_{r}=16$ ) provide performance results close to those for $S_{t}=S_{r}=1$ with $f_{D} T=0.001$ and $f_{D} T=0.005$, respectively. Therefore, although not precise, (5) provides a rough estimate of the achievable effective fade rate, $f_{D} T(2)$, and hence the diversity gain for a given value of $T_{c}$. In addition, (6) estimates the required value of $S_{t} S_{r}$.

So, we have seen that switching reconfigurable antenna states every $T_{c}$ symbol periods to provide independent channels can provide diversity gains, also called antenna state switching gains, and hence the performance expected of a higher fade rate.

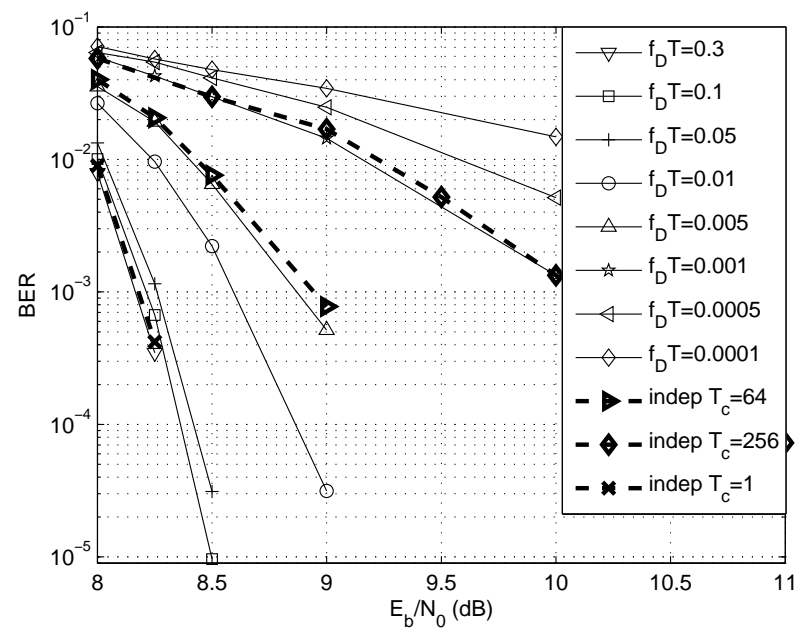

Fig. 3. BER of a $\mathcal{R}_{s t c}=2$ ST-LDPC code using traditional antennas ( $S_{t}=S_{r}=1$ ) versus performance using independent ("indep") channel matrices every $T_{c}=1,64$ or 256 symbol periods. Each antenna transmits points from 16-QAM. The "indep" simulations use $f_{D} T=0.0001$.

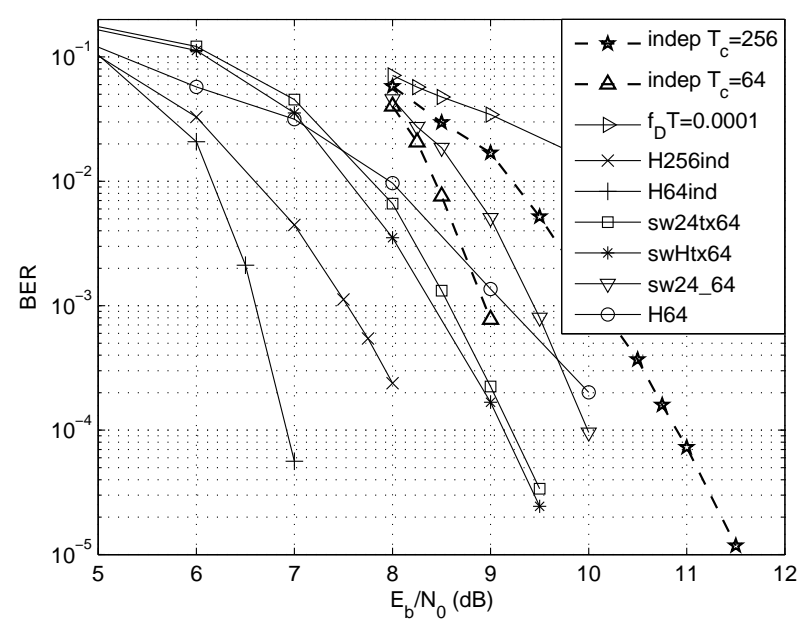

Fig. 4. BER of a $\mathcal{R}_{s t c}=2$ ST-LDPC code using various antenna approaches. Each antenna transmits points from 16-QAM. We use $f_{D} T=0.0001$.

\section{B. Antenna State Selection}

Now we consider selecting the antenna state rather than switching it at predefined times. This provides a more adaptive approach and allows us to achieve significant SNR gains. To this end, some example selection schemes are presented. In these examples, we assume $f_{D} T=0.0001$ and $S_{t}=S_{r}=2$ giving $S_{t} S_{r}=4$ independent channel matrices and a large number of correlated channel matrices. For comparison purposes, we include the transmit antenna state switching scheme of section III.A, which switches to an independent channel every $T_{c}=256$ symbol periods, denoted"indep $T_{c}=256$ " in Fig. 4. The original $f_{D} T=0.0001$ curve is also shown in Fig. 4.

In order to explore the possible SNR gain, also called antenna state selection gain, we consider selecting the antenna states at both the transmitter and receiver. We select the transmit and receive antenna states every $T_{c}=64$ symbol 
periods ${ }^{1}$ according to the channel matrix energy at the start of each block of $T_{c}$ symbol periods and keep the choice fixed over the block. Specifically, we choose the channel matrix with index ${ }^{2}$ [2]

$$
p_{\max }=\arg \max _{p} \sum_{i=1}^{N_{t}} \sum_{j=1}^{N_{r}}\left|h_{t, i, j}(p)\right|^{2},
$$

where $t$ is the symbol period index at the start of the block Note that this requires a feedback channel from receiver to transmitter. This gives the "H64" result in Fig. 4. Although, an SNR gain is achieved, this is at the cost of diversity. This loss in diversity is due to the channel varying sufficiently slowly so that a new value of $p_{\max }$ and hence a new channel matrix is not chosen for every block of $T_{c}=64$ symbol periods.

Now we wish to achieve the diversity offered by the independent channels (seen using antenna state switching) while achieving the SNR gain possible by selecting the best antenna states using (7). To this end, we cycle the transmit antenna states through the $S_{t}^{N_{t}}=16$ possible state combinations over the codeword duration, resulting in a change every $T_{c}=64$ symbol periods. This also means that no receiver to transmitter feedback path is required as the transmit antenna state switching is pre-defined. At the receiver, the receive antenna state (and hence channel matrix) is chosen every $T_{c}=64$ symbol periods using (7). This gives the "swHtx64" result in Fig. 4. As can be seen, the diversity is similar to "indep $T_{c}=256$ ", while achieving approximately $1.75 \mathrm{~dB}$ of SNR gain at $2 \times 10^{-4}$.

In [1], the increase in average receive SNR, from using antenna state selection instead of switching, called the selection gain, was given by

$$
\text { Selection gain }=\frac{\mathrm{E}\left[\left|\boldsymbol{H}\left(p_{\max }\right)\right|^{2}\right]}{P \mathrm{E}\left[\frac{|\boldsymbol{H}(1)|^{4}}{\sum_{p=1}^{P}|\boldsymbol{H}(p)|^{2}}\right]} \geq 1,
$$

where $\mathrm{E}[\cdot]$ denotes the expected value. Using simulation and (8) we found the selection gain for $S_{t}=S_{r}=2, N_{t}=N_{r}=4$ and $P=256$ was approximately 1.5 (or $1.76 \mathrm{~dB}$ ) around a BER of $10^{-4}$. This is close to the SNR gain of approximately $1.75 \mathrm{~dB}$ found in Fig. 4 when comparing the "swHtx64" and "indep $T_{c}=256$ " results at a BER of approximately $2 \times 10^{-4}$. Therefore, (8) also provides a good estimate of the selection gain in the coded case considered here, as would be expected.

Slight diversity gains beyond those shown by "indep $T_{c}=$ 256 " can be achieved by using channel matrices which differ in two rows and two columns in addition to those which differ in all 4 rows/ columns. If we use all 16 of such matrices, one every $T_{c}=64$ symbol periods, then we obtain the "sw24_64" result in Fig. 4. A slight diversity gain is achieved, but as there is no freedom in selecting antenna states, we do not realize the antenna selection SNR gains seen when using "swHtx64". If instead we use only the antenna state combinations at the transmitter to ensure 2 or 4 rows of $\boldsymbol{H}$ change, but allow the receive antenna states to be selected using (7), then "sw24tx64" in Fig. 4 is obtained. It achieves similar diversity to "sw24_64", but provides $0.88 \mathrm{~dB}$ SNR gain at $2 \times 10^{-4}$ BER compared to "sw24_64". Initially "sw24tx64" has worse performance than "swHtx64", but by $2 \times 10^{-4}$ they have similar performance.

The antenna switching gain, assuming $T_{c}=64$, is bounded by what could be achieved if the channel matrix changed independently every $T_{c}=64$ symbol periods, "indep $T_{c}=$ 64 " in Fig. 4. The antenna selection gain is bounded by what could be achieved if all $P$ channel matrices changed independently every 256 symbol periods and we selected $\boldsymbol{H}$ every $T_{c}=64$ symbol periods using (7), called "H256ind" in Fig. 4. For completeness, we also show the result assuming independent channels are generated every 64 symbol periods, called "H64ind". None of these very loose lower bounds are realizable as the actual set of channels lack the independence assumed in these bounding simulations, but they are useful for checking the diversity gains achieved.

\section{CONCLUSION}

In this letter, we consider MIMO transmissions using spacetime codes or error control codes spanning hundreds or thousands of symbol periods. We show that reconfigurable antennas can be used to recover diversity losses due to slow fading through antenna state switching. This makes the codes effective over a much wider range of fade rates even for low complexity (and no feedback) switching schemes. In addition, reconfigurable antennas are shown to provide SNR gains through antenna state selection. By using antenna state switching at the transmitter and antenna state selection at the receiver (e.g. "swHtx64" and "sw24tx64") we keep complexity feasible, avoid having a feedback path and achieve excellent diversity and SNR gains.

\section{REFERENCES}

[1] F. Fazel, A. Grau, H. Jafarkhani and Franco De Flaviis, "Space-timestate block coded MIMO communication systems using reconfigurable antennas," IEEE Trans. Wireless Commun., vol. 8, no. 12, pp. 6019-6029, Dec. 2009

[2] A. Grau, H. Jafarkhani and Franco De Flaviis, "A reconfigurable multiple-input multiple-output communication system," IEEE Trans. Wireless Commun., vol. 7, no. 5, pp. 1719-1733, May 2008.

[3] X.-Y. Hu, E. Eleftheriou, D.-M. Arnold and A. Dholakia, "Efficient implementations of the sum-product algorithm for decoding LDPC Codes," in Proc. Globecom, vol. 2, pp. 25-29, 2001.

[4] X.-Y. Hu, E. Eleftheriou and D.-M. Arnold, "Progressive edge growth Tanner graphs," in Proc. Globecom, vol. 2, pp. 995-1001, 2001.

[5] B. M. Hochwald and S. ten Brink, "Achieving near-capacity on a multiple-antenna channel," IEEE Trans. Commun., vol. 51, no. 3, pp. 389-399, Mar. 2003.

[6] P. A. Martin, D. M. Rankin and D. P. Taylor, "Multi-dimensional spacetime multilevel codes," IEEE. Trans. Wireless Commun., vol. 5, no. 11, pp. 3287-3295, Nov. 2006.

[7] T. Richardson, A. Shokrollahi and R. Urbanke, "Design of capacityapproaching irregular low-density parity check codes," IEEE Trans. Inform. Theory, vol. 47, pp. 619-637, Feb. 2001.

[8] P. Robertson, E. Villebrun and Peter Hoeher, "A comparison of optimal and sub-optimal MAP decoding algorithms operating in the log domain," in Proc. ICC, vol. 2, pp. 1009-1013, 1995.

[9] R. van Nobelen, "Coding for the Rayleigh fading channel", $\mathrm{PhD}$ Thesis, University of Canterbury, Christchurch, New Zealand, 1996.

${ }^{1} T_{c}=64$ is chosen for comparison purposes.

${ }^{2}$ See [1], [2] for more information on antenna selection. 